\title{
Quantificação do volume total por meio de modelos volumétricos e de afilamento em Eucalyptus grandis W. Hill ex Maiden
}

\author{
Quantification of total volume by volumetric models and tapering in Eucalyptus grandis W. Hill ex \\ Maiden
}

Klerysson Julio Farias*, Marcos Felipe Nicoletti \& Natália Saudade de Aguiar

Universidade do Estado de Santa Catarina, Lages, SC, Brasil. *Autor para correspondência: kjfarias.efl@gmail.com.

Submissão: 27/09/2017 / Aceite: 04/04/2018

\begin{abstract}
RESUMO
O presente estudo teve como comparar o desempenho das equações obtidas por meio de modelos volumétricos e de função de afilamento para obtenção do volume total individual com casca em povoamentos de Eucalyptus grandis, em Itatinga, SP. Para tanto, foram cubadas 180 árvores pelo método de Smalian. Após isso, foram selecionados e testados seis modelos volumétricos e quatro modelos de função de afilamento, sendo que para a seleção das melhores equações utilizaram-se os coeficientes de ajuste e precisão: $\mathrm{R}^{2}$ ajustado, erro padrão da estimativa absoluto e em porcentagem e a análise gráfica da dispersão residual. Os melhores modelos de volumetria e afilamento foram submetidos ao teste qui-quadrado, a um nível de significância com $5 \%$ de probabilidade de erro, para verificar se existem diferenças significativas nas estimativas das equações. As equações volumétricas e de afilamento apresentaram bons valores de ajuste, grande maioria com $\mathrm{R}^{2}$ ajustado maior que 0,95 e erros inferiores a $13 \%$. As equações obtidas por meio do modelo de Meyer e do modelo Polinomial de $5^{\circ}$ grau apresentaram os melhores desempenhos, sendo que após a análise pelo teste qui-quadrado ambos obtiveram valores calculados inferiores ao tabelado, indicando que tanto um quanto outro podem ser utilizados para estimativa do volume total das árvores.
\end{abstract}

PALAVRAS-CHAVE: análises estatísticas com R, produção florestal, cubagem, igualdade estatística entre modelos.

\begin{abstract}
This study had as objective comparing the performance of equations obtained by means of volumetric models and tapering function to obtain the total individual volume with bark of Eucalyptus grandis, in Itatinga, SP. For such, 180 trees were measured through the Smalian method. After that, six volumetric and four tapering functions were selected and tested, and to select the equations that provided the best adjustment indices, adjustment and precision coefficients were used: adjusted $\mathrm{R}^{2}$, standard error of the absolute estimate, as well as percentage and graphical analysis of the residual dispersion. The best volumetric models and tapering functions were subjected to the chi-square test at a significance level of $5 \%$ to verify if there was significant difference in the estimates of the equations. The volumetric and tapering function equations presented good adjustment values, a large majority with $\mathrm{R}^{2}$ adjusted higher than 0.95 while having errors lower than $13 \%$. The equations obtained using the Meyer model and the Polynomial model of the 5th degree showed the best performances, and after the chi-square test, both obtained lower values than the table, indicating that both can be used to estimate the total volume of trees.
\end{abstract}

KEYWORDS: R statistical analysis, forest production, measurement, equality of models.

\section{INTRODUÇÃO}

Dentre as informações obtidas da floresta, o volume destaca-se devido fornecer um ponto de partida para a avaliação do estoque de madeira e análise do potencial produtivo (MACHADO et al. 2004). Este pode ser obtido por diferentes métodos, como cubagem, estimativas por equações de volume, fatores de forma, funções de afilamento, entre outros, buscando a determinação precisa da variável de interesse.

As equações volumétricas são amplamente utilizadas para estimativas de volume, pela relativa facilidade e acurácia, sendo ajustadas para apresentar os menores erros possíveis. De acordo com GUIMARÃES \& LEITE (1996) a utilização de equações volumétricas se constitui do procedimento mais eficiente para a 
quantificação da produção florestal.

O procedimento é realizado pela cubagem rigorosa de algumas árvores para a determinação dos volumes, os quais, por meio de equações de regressão são estimados em função de variáveis dendrométricas. Podem ser utilizados modelos de simples ou dupla entrada, sendo que os de simples entrada utilizam como variável independente o diâmetro, e os de dupla entrada o diâmetro e a altura, ou uma combinação destes (SCHNEIDER 1997).

Já os modelos de afilamento são muito eficazes quando se busca o direcionamento da produção de uma floresta para multiprodutos, devido a capacidade de identificar as diferentes potencialidades que a árvore oferece (TÉO et al. 2013). De acordo com NICOLETTI (2017), o emprego de funções de afilamento proporciona uma otimização dos recursos florestais, e segundo FISCHER et al. (2001) permitem avaliar biológica e economicamente o maciço florestal e a resposta às práticas de manejo executadas, já que permitem valorar de maneira detalhada quanto renderá o povoamento florestal.

Dessa forma, em consequência da sua flexibilidade e grande número de informações que proporcionam sem aumento dos custos de inventário, as funções de afilamento têm ganhado espaço no meio florestal (ASSIS et al. 2002). Muitos modelos de taper foram desenvolvidos desde a primeira proposta feita pelo sueco Hojer em 1903, como modelos simples, segmentados, polinomiais e sigmoides (ANDRADE 2014).

Esses modelos têm capacidade de descrição matemática do tronco, devido a forma do fuste não se identificar com um único sólido específico em função de seu afilamento. Os modelos de afilamento são funções que representam a variação diamétrica ao longo do fuste da árvore (PRODAN et al. 1997), e assim de acordo com MACHADO et al. (2004), permitem a estimativa do diâmetro numa altura qualquer do fuste, da altura comercial para determinado diâmetro superior, e segundo AHRENS \& HOLBERT (1981) o volume pode ser obtido por integração, sendo tratado como um sólido de revolução.

Ante ao exposto, devido a importância da estimativa do volume de um povoamento florestal, e buscando métodos com a maior precisão possível, o presente estudo teve como objetivo comparar as estimativas obtidas por meio de modelos volumétricos e de afilamento para obtenção do volume total individual em povoamentos de Eucalyptus grandis, em Itatinga, SP.

\section{MATERIAL E MÉTODOS}

O estudo foi realizado na Estação Experimental de Ciências Florestais de Itatinga do Departamento de Ciências Florestais da Universidade de São Paulo (ESALQ/USP), localizada na região Centro Sul do Estado de São Paulo, com 857 metros de altitude média. O clima da região, segundo Köppen, é caracterizado como mesotérmico úmido (Cwa), com precipitação anual média de $1.308 \mathrm{~mm}$. A temperatura média do mês mais frio $12,8 \stackrel{\circ}{\circ} \mathrm{C}$ e a média do mês mais quente de 19,4 으 (NICOLETTI et al. 2015). As medidas de campo foram realizadas em um experimento de Eucalyptus grandis Maiden com 13 anos de idade e espaçamento $3 \mathrm{~m} \times 2 \mathrm{~m}$, sem desbaste.

Foram cubadas 180 árvores pelo método de Smalian, nas posições 0,$10 ; 0,70 ; 1,30$ metros e a partir desta de metro em metro, sendo que, após a coleta dos dados, foram selecionados e testados seis modelos volumétricos e quatro modelos de afilamento (Tabela 1 ).

O modelo de Hradetzky da Tabela 1 encontra-se apresentado no formato anterior a seleção das variáveis independentes no procedimento de Stepwise (STEPKA et al. 2017). Todos os ajustes foram desenvolvidos por meio de regressão linear, não linear e Stepwise no software $R$ ( $R$ DEVELOPMENT CORE TEAM 2017).

Para a obtenção do volume total individual para os modelos de afilamento realizou-se a integração destes segundo MIGUEL et al. (2011), sendo que o resultado dessa operação para o modelo de GARCIA et al. (1993) é apresentado na Equação 1:

$$
\mathrm{v}=\mathrm{K} * \mathrm{~d}^{2} *\left[\beta_{0} \mathrm{~h}+\frac{2 \beta_{1}\left(\frac{\mathrm{hi}}{\mathrm{h}}\right)^{\frac{\varepsilon}{2}} \mathrm{~h}}{3}+\frac{\beta_{2} \mathrm{~h}^{2}}{2 \mathrm{~h}}\right]
$$

(Equação 1)

Em que: $v=$ volume total com casca $\left(\mathrm{m}^{3}\right) ; \mathrm{K}=\left(\frac{\pi}{40,000}\right) ; \mathrm{d}^{2}=$ diâmetro a $1,30 \mathrm{~m}$ do solo, elevado ao quadrado $(\mathrm{cm})^{2} ; \beta_{n}=$ Coeficientes da regressão; $\mathrm{h}_{\mathrm{i}}=$ Altura na posição $\mathrm{i}(\mathrm{m}) ; \mathrm{h}=$ Altura total $(\mathrm{m})$. 
Tabela 1. Modelos volumétricos e funções de afilamento utilizados para os ajustes na população de Eucalyptus grandis.

Table 1. Volumetric models and tapering functions used for adjustments in the population of Eucalyptus grandis.

\begin{tabular}{|c|c|c|}
\hline & Autor & Modelo empírico \\
\hline \multirow{6}{*}{ Volumetria } & Berkhout & $\mathrm{v}=\beta 0 * \mathrm{~d}^{\beta 1}$ \\
\hline & $\begin{array}{l}\text { I.B.W } \\
\text { Alemanha }\end{array}$ & $\ln (\mathrm{v})=\beta 0+\beta 1 * \ln (\mathrm{d})+\beta 2 * \ln ^{2}(\mathrm{~d})+\beta 3 * \ln (\mathrm{h})+\beta 4 * \ln ^{2}(\mathrm{~h})+$ ei \\
\hline & Meyer & $v=\beta 0+\beta 1 * d+\beta 2 * d^{2}+\beta 3 * d h+\beta 4 * d^{2} h+e i$ \\
\hline & Prodan I & $\sqrt{v}=\beta 0+\beta 1 * d h+\beta 2 * d^{2} h^{2}+\beta 3 * d^{3} h^{3}+\beta 4 * d^{4} h^{4}+\beta 5 * d^{5} h^{5}+\beta 6 * d^{6} h^{6}+e i$ \\
\hline & $\begin{array}{l}\text { Schumach } \\
\text { er Hall }\end{array}$ & $\ln (v)=\beta 0+\beta 1 * \ln (d)+\beta 2 * \ln (h)+e i$ \\
\hline & Spurr & $v=\beta 0+\beta 1 * d^{2} h+e i$ \\
\hline \multirow{4}{*}{ Afilamento } & $\begin{array}{l}\text { Garcia et } \\
\text { al. (1993) }\end{array}$ & $y^{2}=\beta 0+\beta 1 * \sqrt{x}+\beta 2 * x+e i$ \\
\hline & $\begin{array}{l}\text { Hradetzky } \\
\text { (1979) }\end{array}$ & $y=\beta 0+\beta 1 * x^{p 1}+\beta 2 * x^{p 2}+\beta 3 * x^{p 3}+\cdots+\beta_{n-1} * x^{p n-1}+\beta_{n} * x^{p m}+e i$ \\
\hline & $\begin{array}{l}\text { Kozak et } \\
\text { al. (1969) }\end{array}$ & $y^{2}=\beta 0+\beta 1 * x+\beta 2 * x^{2}+e i$ \\
\hline & $\begin{array}{l}\text { Schoepfer } \\
(1966)\end{array}$ & $y=\beta 0+\beta 1 * x+\beta 2 * x^{2}+\beta 3 * x^{3}+\beta 4 * x^{4}+\beta 5 * x^{5}+e i$ \\
\hline
\end{tabular}

Em que: $v=$ volume total individual $\left(\mathrm{m}^{3}\right) ; \mathrm{d}=$ diâmetro a $1,30 \mathrm{~m}$ do solo $(\mathrm{cm}) ; \mathrm{h}=$ altura total $(\mathrm{m}) ; \mathrm{d}_{\mathrm{i}}=$ diâmetro na posição i ao longo do fuste da árvore $(\mathrm{cm}) ; h_{i}=$ altura na posição $i$ ao longo do fuste da árvore $(\mathrm{m}) ; \mathrm{y}=\mathrm{d}_{\mathrm{i}} / \mathrm{d} ; \mathrm{x}=\mathrm{h}_{\mathrm{i}} / \mathrm{h} ; \beta_{\mathrm{n}}=$ coeficientes do modelo; pi = expoentes variando entre 0,005 e 25; In = Logaritmo neperiano. Fonte: MIGUEL et al. (2011), SILVA et al. (2011), SILVESTRE et al. (2014).

A integração do modelo de potências fracionárias (Hradetzky) após a análise por Stepwise foi dada pela Equação 2:

$$
\begin{aligned}
& \mathrm{v}=\mathrm{K} * \mathrm{~d}^{2} *\left[\mathrm{c}_{0}^{2} \mathrm{hi}+2 \mathrm{c}_{0} \mathrm{c}_{1}\left(\frac{\mathrm{hj}^{\left(\mathrm{p}^{1+1}\right)}}{\mathrm{p}_{1}+1}\right)+2 \mathrm{c}_{0} \mathrm{c}_{2}\left(\frac{\mathrm{hi}^{\left(\mathrm{p}^{2+1}\right)}}{\mathrm{p}_{2}+1}\right)+\cdots+2 \mathrm{c}_{0} \mathrm{c}_{(\mathrm{n}-1)}\left(\frac{\mathrm{hi}^{(\mathrm{p}(\mathrm{n}-1)+1)}}{\mathrm{p}_{(\mathrm{n}-1)}+1}\right)+\right.
\end{aligned}
$$

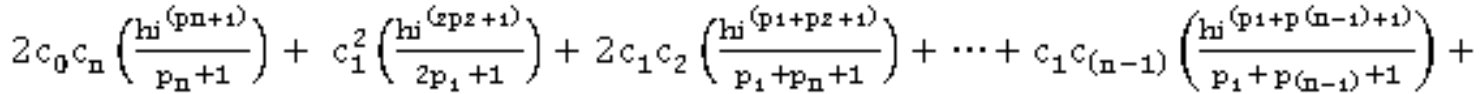

$$
\begin{aligned}
& 2 c_{1} c_{n}\left(\frac{h^{\left(p^{(1+p n+1)}\right.}}{p_{1}+p_{n}+1}\right)+c_{2}^{2}\left(\frac{h^{\left(p^{2}+1\right)}}{2 p_{2}+1}\right)+\cdots+2 c_{2} c_{(n-1)}\left(\frac{h^{\left(p^{2}+p^{(n-1)+1)}\right.}}{p_{2}+p_{n}+1}\right)+2 c_{2} c_{n}\left(\frac{h^{\left(p^{2}+p^{n+1}\right)}}{p_{2}+p_{n}+1}\right)+ \\
& \left.\cdots+c_{(n-1)}^{2}\left(\frac{h^{\left(2 p^{(n-1)+1)}\right.}}{2 p(n-1)}\right)+\ldots+2 c_{(n-1)} c_{n}\left(\frac{h^{(p(n-1)+p n+1)}}{p_{(n-1)}+p_{n}+1}\right)+c_{n}^{2}\left(\frac{h^{(2 p n+1)}}{2 p_{n}+1}\right)\right]_{h 1}^{h 2}
\end{aligned}
$$

Em que: $v=$ volume total com casca $\left(\mathrm{m}^{3}\right) \mathrm{K}=\left(\frac{\pi}{40.000}\right) ; \mathrm{d}^{2}=$ diâmetro a $1,30 \mathrm{~m}$ do solo, elevado ao quadrado $(\mathrm{cm})^{2} ; c_{0}=\beta_{0} ; c_{1}=\frac{\beta_{1}}{h_{h}} ; c_{2}=\frac{\beta_{2}}{h^{2}} ; \ldots . ;{ }^{c}=\frac{\beta_{n}}{h^{2}} ; h_{i}=$ altura na posição $\mathrm{i}(\mathrm{m}) ; \mathrm{pn}=$ expoentes do modelo de Hradetzky selecionados na Stepwise.

Já para KOZAK et al. (1969), a estimativa do volume total após a integração da equação de função de afiamento é dada pela Equação 3:

$$
\mathrm{v}=\mathrm{K} * \mathrm{~d}^{2} *\left[\beta_{0}\left(\mathrm{~h}_{2}-\mathrm{h}_{1}\right)+\frac{\beta_{1}}{2 \mathrm{~h}}\left(\mathrm{~h}_{2}^{2}-\mathrm{h}_{1}^{2}\right)+\frac{\beta_{2}}{3 \mathrm{~h}^{2}}\left(\mathrm{~h}_{2}^{3}-\mathrm{h}_{1}^{3}\right)\right]
$$

(Equação 3)

Em que: $v=$ volume total com casca $\left(\mathrm{m}^{3}\right) ; \mathrm{K}=\left(\frac{\pi}{40.000}\right) ; \mathrm{d}^{2}=$ diâmetro a $1,30 \mathrm{~m}$ do solo, elevado ao quadrado $(\mathrm{cm})^{2} ; \beta_{n}=$ Coeficientes da regressão; $\mathrm{h}_{2}=$ Altura total $(\mathrm{m}) ; \mathrm{h}_{1}=$ Altura na posição $\mathrm{i}(\mathrm{m}) ; \mathrm{h}=$ Altura total $(\mathrm{m})$.

O polinômio de $5^{\circ}$ grau de SCHOEPFER (1966) apresenta a seguinte equação de estimativa para o volume total (Equação 4): 


$$
\begin{aligned}
& \mathrm{v}=\mathrm{K} * \mathrm{~d}^{2} *\left[c_{0}^{2} \mathrm{hi}+\mathrm{c}_{0} \mathrm{c}_{1} \mathrm{hi}^{2}+\left(\frac{2}{3} c_{0} c_{2}+\frac{1}{3} c_{1}^{2}\right) h \mathrm{i}^{3}+\left(\frac{1}{2} c_{0} c_{3}+\frac{1}{2} c_{1} c_{2}\right) h i^{4}+\right. \\
& \left(\frac{2}{5} c_{0} c_{4}+\frac{2}{5} c_{1} c_{3}+\frac{1}{5} c_{2}^{2}\right) h i^{5}+\left(\frac{1}{3} c_{0} c_{5}+\frac{1}{3} c_{1} c_{4}+\frac{1}{3} c_{2} c_{3}\right) h i^{6}+\left(\frac{2}{7} c_{1} c_{5}+\frac{2}{7} c_{2} c_{4}+\right. \\
& \left.\left.\frac{1}{7} c_{3}^{2}\right) h i^{7}+\left(\frac{1}{4} c_{3} c_{5}+\frac{1}{4} c_{3} c_{4}\right) h i^{8}+\left(\frac{2}{9} c_{3} c_{5}+\frac{1}{9} c_{4}^{2}\right) h i^{9}+\frac{1}{5} c_{4} c_{5} h i^{10}+\frac{1}{11} c_{5}^{2} h i^{11}\right]_{h 1}^{h 2}
\end{aligned}
$$

Em que: $v=$ volume total com casca $\left(\mathrm{m}^{3}\right) ; \mathrm{K}=\left(\frac{\pi}{40.000}\right) ; \mathrm{d}^{2}=$ diâmetro a $1,30 \mathrm{~m}$ do solo, elevado ao quadrado $(\mathrm{cm})^{2} ; c_{0}=\beta_{0} ;{ }_{1}=\frac{\beta_{1}}{h} ; c_{2}=\frac{\beta_{2}}{h^{2}} ; \ldots ;{ }^{c_{n}}=\frac{\beta_{\mathrm{n}}}{h^{2}} ; h_{i}=$ altura na posição $\mathrm{i}(\mathrm{m}) ; \mathrm{h}=$ altura total $(\mathrm{m})$.

Para a seleção das equações que forneceram as melhores estimativas para os modelos estudados foram calculados, segundo SILVESTRE et al. (2014), os seguintes parâmetros de indicativa de ajuste: $\mathrm{R}^{2}$ ajustado (Equação 5), erro padrão da estimativa absoluto (Equação 6) e em porcentagem (Equação 7) e a análise gráfica da dispersão residual.

$$
\mathrm{R}_{\text {ajustado }}^{2}=\frac{\mathrm{n}-1}{\mathrm{n}-\mathrm{p}} * \frac{\text { sQresiduo }}{\text { SQtotal }}
$$

\section{(Equação 5)}

Em que: $R^{2}$ ajustado = Coeficiente de determinação ajustado; $n=$ número de observações; $p=$ número de variáveis independentes do modelo; $\mathrm{SQresíduo} \mathrm{=} \mathrm{Soma} \mathrm{do} \mathrm{quadrado} \mathrm{dos} \mathrm{resíduos;} \mathrm{SQtotal} \mathrm{=} \mathrm{Soma} \mathrm{do}$ quadrado total.

$$
\mathrm{S}_{\mathrm{yx}}\left(\mathrm{m}^{3}\right)=\sqrt{\frac{\sum_{i=1}^{n}(y i-\hat{y})^{2}}{n-p}}
$$

Em que: Syx $\left(m^{3}\right)=$ Erro padrão da estimativa absoluto $\left(m^{3}\right) ;$ yi $=$ valor observado $\left(m^{3}\right) ; \hat{y} i=$ valor estimado $\left(\mathrm{m}^{3}\right)$.

$$
S_{y x}(\%)=\left(\frac{s_{y x}}{\bar{y}}\right) * 100
$$

\section{(Equação 7)}

Em que: Syx $(\%)=$ Erro padrão da estimativa em porcentagem; Syx = erro absoluto da estimativa $\left(\mathrm{m}^{3}\right) ; \bar{y}$ $=$ volume médio observado $\left(\mathrm{m}^{3}\right)$.

Os modelos logaritmizados obtiveram suas estimativas recalculadas por meio do Fator de Meyer (MACHADO et. 2008), o qual segundo SILVA et al. (2011) objetiva a correção das estimativas dos modelos que sofreram uma transformação matemática na variável dependente, sendo que apenas o cálculo do antilog em modelos logarítmicos não é suficiente para a minimização desses erros, sendo isso chamado de discrepância logarítmica (Equação 8).

$$
F M=e^{(Q M \text { residuo*0,5) }}
$$

Em que: $\mathrm{FM}=$ Fator de Meyer; $\mathrm{QM}$ resíduo = Quadrado Médio do resíduo.

O melhor modelo de volumetria e o melhor de função de afilamento foram submetidos ao teste qui quadrado, para verificar a eficiência das duas metodologias preditivas, a um nível de significância de $5 \%$ de probabilidade de erro, sendo que as hipóteses foram as seguintes:

H0: Não houve diferença significativa na estimativa do volume por meio da equação volumétrica ou de função de afilamento. de afilamento.

H1: Houve diferença significativa na estimativa do volume por meio da equação volumétrica ou de função

\section{RESULTADOS}

A população analisada obteve um DAP médio de $15,5 \mathrm{~cm}$, com valor mínimo de $10,0 \mathrm{~cm}$ e um valor máximo de $20,8 \mathrm{~cm}$. Já a altura variou de 15,7 a $28,6 \mathrm{~m}$, com média de $25 \mathrm{~m}$. Os volumes obtiveram valores de 0,0587 a 0,4604 , com média de 0,2331 m. Diante disso, realizaram-se os ajustes dos modelos matemáticos, sendo que seus resultados estão apresentados na Tabela 2.

Observou-se que para as equações obtidas com os modelos volumétricos selecionados (Tabela 3) o valor do $R^{2}$ ajustado foi de 0,9348 a 0,9625 . O erro padrão da estimativa absoluto variou de 0,0156 a $0,0205 \mathrm{e}$ o erro padrão da estimativa relativo de $6,7 \%$ até $10,8 \%$, cerca de $38,5 \%$ de variação entre o menor e o maior valor. 
Tabela 2. Estatísticas de ajuste e precisão dos modelos volumétricos e de afilamento para a população de Eucalyptus grandis analisada.

Table 2. Adjustment and accuracy statistics of the volumetric and tapering models for the population of Eucalyptus grandis analyzed.

\begin{tabular}{|c|c|c|c|c|c|}
\hline Modelo & Coeficientes & FM & $\mathrm{R}^{2}$ ajustado & Syx $\left(m^{3}\right)$ & Syx (\%) \\
\hline \multirow[t]{2}{*}{ Berkhout } & $\beta_{0}=0,00032$ & 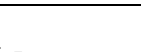 & 09348 & & 88087 \\
\hline & $\beta_{1}=2,37201$ & & 0,9348 & $0,0<05$ & $8,808 /$ \\
\hline \multirow[t]{7}{*}{ I.B.W. Alemanha } & $\beta_{0}=-6,9703$ & 1,00212 & 0,9623 & 0,0156 & 6,6991 \\
\hline & $\beta_{1}=1,82073$ & & & & \\
\hline & $\beta_{2}=0,02205$ & & & & \\
\hline & $\beta_{3}=-0,87636$ & & & & \\
\hline & $\beta_{4}=0,30284$ & & & & \\
\hline & $\beta_{0}=-0,07912$ & - & 0,9625 & 0,0156 & 6,6754 \\
\hline & $\beta_{1}=0,02553$ & & & & \\
\hline \multirow[t]{2}{*}{ Meyer } & $\beta_{2}=-0,00144$ & & & & \\
\hline & $\begin{array}{l}\beta_{3}=-0,00062 \\
\beta_{4}=0,00013\end{array}$ & & & & \\
\hline \multirow[t]{7}{*}{ Prodan I } & $\beta_{0}=0,06944$ & - & 0,9625 & 0,0156 & 6,6795 \\
\hline & $\beta_{1}=0,00123$ & & & & \\
\hline & $\beta_{2}=0,00001$ & & & & \\
\hline & $\beta_{3}=-1,83982$ & & & & \\
\hline & $\beta_{4}=5,32374$ & & & & \\
\hline & $\beta_{5}=-3,7520$ & & & & \\
\hline & $\beta_{6}=0,00001$ & & & & \\
\hline \multirow[t]{3}{*}{ Schumacher - Hall } & $\beta_{0}=-10,11512$ & 1,00412 & 0,9623 & 0,0156 & 6,6926 \\
\hline & $\beta_{1}=1,94682$ & & & & \\
\hline & $\beta_{2}=1,02063$ & & & & \\
\hline \multirow[t]{2}{*}{ Spurr } & $\beta_{0}=0,00212$ & - & 0,9625 & 0,0156 & 6,6778 \\
\hline & $\beta_{1}=0,00001$ & & & & \\
\hline \multirow[t]{3}{*}{ Garcia et al. (1993) } & $\beta_{0}=1,5359$ & - & 0,9524 & 0,0196 & 8,3021 \\
\hline & $\beta_{1}=-1,9684$ & & & & \\
\hline & $\beta_{2}=0,5608$ & & & & \\
\hline \multirow[t]{9}{*}{ Hradetzky } & $\beta_{0}=-9477,47301$ & - & 0,9554 & 0,0169 & 7,7283 \\
\hline & $\beta_{0,008}=11751,86202$ & & & & \\
\hline & $\beta_{0,09}=-14283,94820$ & & & & \\
\hline & $\beta_{0,1}=12028,71234$ & & & & \\
\hline & $\beta_{1}=-36,7245$ & & & & \\
\hline & $\beta_{2}=45,39699$ & & & & \\
\hline & $\beta_{3}=-55,07633$ & & & & \\
\hline & $\beta_{4}=39,000592$ & & & & \\
\hline & $\beta_{5}=-11,56483$ & & & & \\
\hline \multirow[t]{3}{*}{ Kozak et al. (1969) } & $\beta_{0}=1,24662$ & - & 0,9008 & 0,0253 & 10,8908 \\
\hline & $\beta_{1}=-2,30442$ & & & & \\
\hline & $\beta_{2}=1,27333$ & & & & \\
\hline \multirow[t]{6}{*}{ Polinômio 5 grau } & $\beta_{0}=1,22093$ & - & 0,9602 & 0,016 & 6,8824 \\
\hline & $\beta_{1}=-4,574741$ & & & & \\
\hline & $\beta_{2}=22,32712$ & & & & \\
\hline & $\beta_{3}=-53,82902$ & & & & \\
\hline & $\beta_{4}=57,82932$ & & & & \\
\hline & $\beta_{5}=-23,01303$ & & & & \\
\hline
\end{tabular}

Em que: $\beta_{n}=$ Coeficientes da regressão; $R^{2}$ ajustado $=$ Coeficiente de determinação ajustado; Syx $=$ Erro padrão da estimativa absoluto $\left(m^{3}\right)$ e Syx $(\%)=$ Erro padrão da estimativa relativo (\%); FM = Fator de correção de Meyer.

Os modelos volumétricos obtiveram valores acima de $96 \%$ de ajuste e erro padrão da estimativa relativo inferior a 7\%, excluindo-se o primeiro (Berkhaut), o qual foi ajustado por meio de regressão não linear, o que 
pode explicar o valor de seus coeficientes de ajuste serem mais baixos que os demais modelos volumétricos.

Berkhout

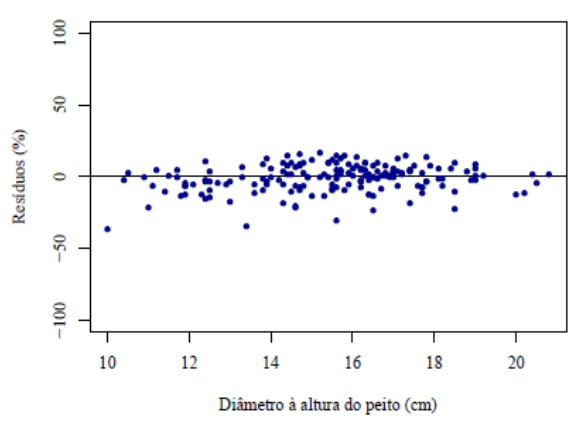

Meyer

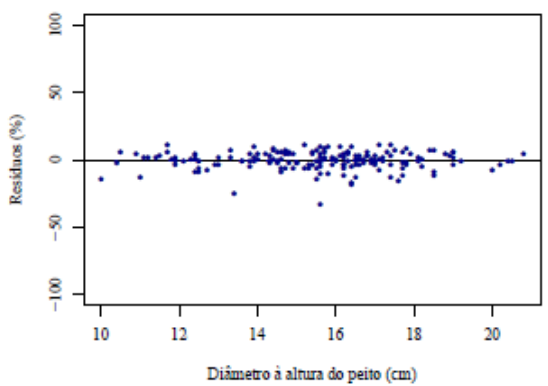

Schumacher Hall

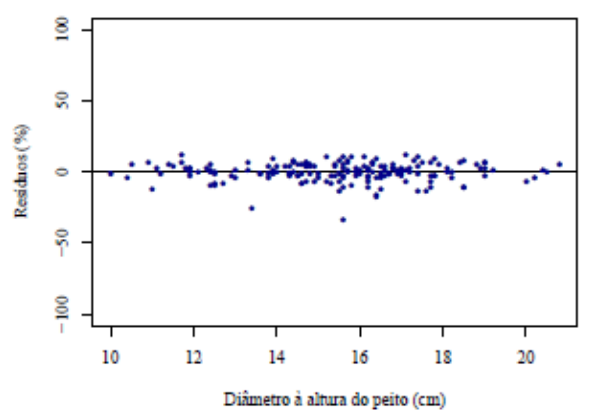

Garcia et al.

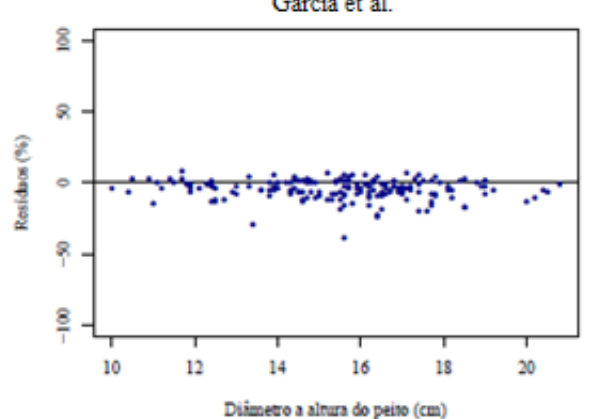

Kozak

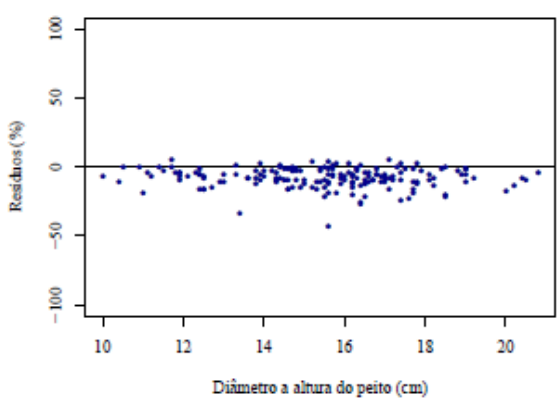

I.B.W Alemanha

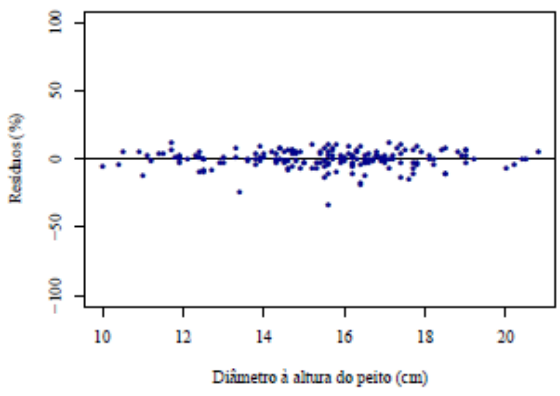

Prodan I

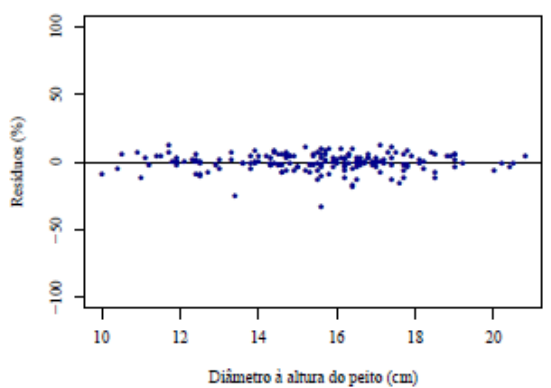

Spurr

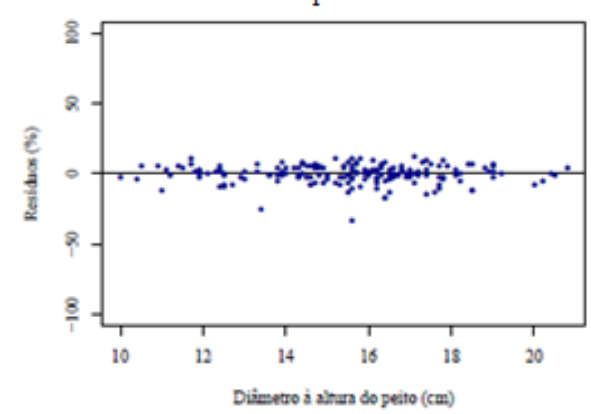

Hradetzky

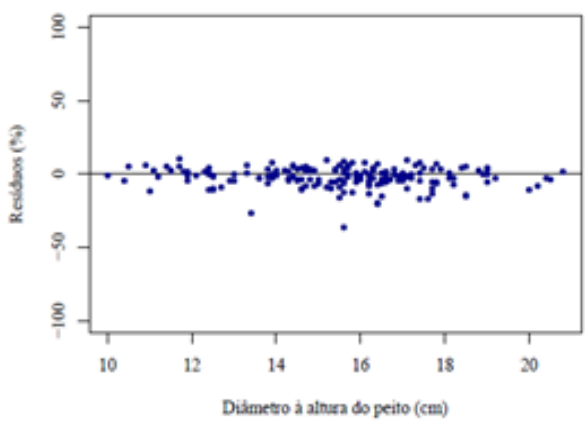

Schoepfer

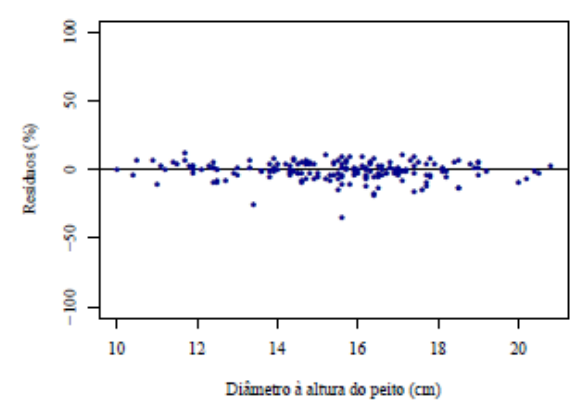

Figura 1. Dispersão residual dos modelos volumétricos e dos modelos de afilamento do povoamento de Eucalyptus grandis.

Figure 1. Residual dispersion of volumetric models and tapering functions of Eucalyptus grandis. 
Os modelos de Meyer, Prodan I e Spurr apresentaram dispersão residual semelhante (Figura 1), sem tendenciosidade nas estimativas. Diante disso, por meio da análise dos coeficientes estatísticos, o modelo que obteve melhor resultado foi o de Meyer, diferindo apenas no erro padrão da estimativa relativo, na terceira casa decimal.

Os modelos de afilamento para estimativa do volume total também obtiveram bons resultados, com valores de ajuste do coeficiente de determinação ajustado de $95 \%$ e erro padrão da estimativa relativo inferior a $9 \%$, excluindo-se o modelo de KOZAK et al. (1969), o qual apresentou menor desempenho.

Analisando-se a dispersão residual dos modelos de afilamento (Figura 1), HRADETZKY (1976) e o polinômio do $5^{\circ}$ grau apresentaram melhores estimativas, sendo que o primeiro gerou uma leve tendência em superestimar os valores de DAP entre 14 e $16 \mathrm{~cm}$. Comparando os coeficientes estatísticos, observou-se que o polinômio de $5^{\circ}$ grau apresentou melhores resultados, apesar de levar em conta menos variáveis no ajuste.

Apesar disso, analisando-se os valores obtidos no teste qui quadrado, a equação do modelo de Meyer apresentou um valor de $X^{2}$ calculado de 0,1609 e o Polinomial de $5^{\circ}$ grau apresentou 0,1667. Ambos foram menores que o valor encontrado no tabelado, de 211,21 para a situação em questão. Desse modo, foi aceita a hipótese de nulidade em ambos os casos, ou seja, os volumes estimados por ambas as equações e o volume obtido na cubagem rigorosa por Smalian não diferiram entre si a $5 \%$ de probabilidade de erro.

\section{DISCUSSÃO}

Os modelos volumétricos apresentados nesse estudo demonstraram ajustes melhores que os encontrados por AZEVEDO et al. (2011), os quais analisaram o desempenho de modelos volumétricos em Eucalyptus sp. em Sergipe. O modelo que obteve o melhor desempenho para os autores foi Schumacher Hall, com um coeficiente de determinação ajustado de 0,845 e um erro padrão relativo acima dos $10 \%$.

Em relações volumétricas, normalmente o valor do coeficiente de determinação ajustado demonstra-se acima de 0,9, como observaram SILVESTRE et al. (2014) e PEREIRA et al. (2016) e como foi apresentado nesse estudo.

Analisando-se as equações obtidas da integração dos modelos de função de afilamento, KOZAK et al. (1969), obteve o pior desempenho. MIGUEL et al. (2011) analisando modelos de afilamento para estimativa do volume total do fuste em Eucalyptus urophylla também obteve como pior modelo Kozak. Já HRADETZKY (1976) obteve uma leve vantagem nos coeficientes estatísticos comparado com o Polinômio do 5o grau, entretanto, quando comparadas as dispersões residuais dos modelos o autor observou que HRADETZKY (1976) possuiu uma ligeira tendência de superestimar os valores, bem como neste estudo.

O polinômio de $5^{\circ}$ grau, no estudo de MIGUEL et al. (2011) apresentou melhores estimativas quando comparado com os demais modelos de afilamento para estimativa do volume total. SILVA et al. (2011) obtiveram resultados diferentes, sendo que para os autores o melhor modelo para estimativa do volume total em Pinus caribaea var. hondurensis o polinômio de potências fracionárias (HRADETZKY 1976). Analisando-se os coeficientes estatísticos de ajuste e as dispersões residuais observou-se que os modelos volumétricos forneceram melhores resultados. STEPKA et al. (2017) realizando estudo semelhante em Pinus taeda L. no Paraná também concluíram que as equações volumétricas demonstraram maior eficiência no ajuste de todo o conjunto de dados, com erros inferiores a $10 \%$ e coeficientes de determinação acima de 0,9 .

\section{CONCLUSÃO}

A maior parte dos modelos analisados possuiu um erro relativo inferior a $10 \%$ e os melhores resultados dos coeficientes estatísticos de ajuste e precisão foram obtidos pelos modelos volumétricos.

Analisando-se os dois melhores modelos da volumetria e afilamento, observou-se que o modelo volumétrico obteve melhores resultados, entretanto, quando comparados por meio do teste qui-quadrado as estimativas obtidas com as equações dos modelos não diferiram entre os valores obtidos na cubagem, indicando que ambos podem ser utilizados para estimativa do volume total individual da população.

Este estudo pode gerar uma base de comparação para outros, sendo que este assunto é de grande valia devido ser base para estimativas de produção e geração de cenários no setor empresarial do mercado florestal.

\section{REFERÊNCIAS}

AHRENS S \& HOLBERT D. 1981. Uma função para forma de tronco e volume de Pinus taeda L. Boletim de Pesquisa Florestal 3: 37- 68.

ANDRADE VCL. 2014. Modelos de taper do tipo expoente-forma para descrever o perfil do fuste de árvores. Pesquisa Florestal Brasileira 34: 271- 283. 
ASSIS AL et al. 2002. Avaliação de modelos polinomiais não-segmentados na estimativa de diâmetros e volumes comerciais de Pinus taeda. Ciência Florestal 12: 89-107.

AZEVEDO TL et al. 2011. Equações hipsométricas e volumétricas para um povoamento de Eucalyptus sp. localizado na FLONA do Ibura, Sergipe. Revista Brasileira de Ciências Agrárias 6: 105-112.

FISCHER F et al. 2001. Exatidão dos modelos polinomiais não-segmentados e das razões entre volumes para representar o perfil do tronco de Pinus taeda. Ciência Florestal 11: 167-188.

GARCIA SLR et al. 1993. Análise do perfil do tronco de morototó (Didymopamax morototonii) em função do espaçamento. In: 1 Congresso Florestal Panamericano, 7 Congresso Florestal Brasileiro. Anais... Curitiba: SBS/SBEF. p.485-491.

GUIMARÃES DP \& LEITE HG. 1996. Influência do número de árvores na determinação de equação volumétrica para Eucalyptus grandis. Scientia Forestalis 50: 37-42.

HRADETZKY J. 1976. Analyse und interpretation statistisher abräanger Keiten. (Biometrische Beiträge zu aktuellen forschungs projekten). Baden: Württemberg Mitteilungen der FVA. 146p. (Abt. Biometric und Informatik, 21).

KOZAK A et al. 1969. Taper functions and their applications in Forest inventory. Forestry Chronicle 45: 278-283.

MACHADO SA et al. 2008. Comportamento da relação hipsométrica de Araucaria angustifolia no capão da Engenharia Florestal da UFPR. Pesquisa Florestal Brasileira 56: 5-16.

MACHADO SA et al. 2004. Comparação de modelos de afilamento do tronco para diferentes idades e regimes de desbaste em plantações de Pinus oocarpa Schiede. Boletim de Pesquisa Florestal 48: 41-64.

MIGUEL EP et al. 2011. Modelos polinomiais para representar o perfil e o volume do fuste de Eucalyptus urophylla na região norte do estado de goiás. Revista Floresta 41: 355-368.

NICOLETTI MF et al. 2015. Exatidão de dendrômetros ópticos para determinação do volume de árvores em pé. Ciência Florestal 25: 395- 404.

NICOLETTI MF. 2017. Modelagem não linear mista e método bivariado para sortimento da produção de Pinus taeda L. Tese (Doutorado em Engenharia Florestal). Curitiba: UFPR. 118p.

PEREIRA ARS et al. 2016. Modelagem volumétrica para Eucalyptus urograndis no Município de Porto Grande, Amapá, Brasil. Biota Amazônia 6: 10-14.

PRODAN M et al. 1997. Mensura Florestal. San Jose: IICA. 586p.

R DEVELOPMENT CORE TEAM. 2017. R: A language and environment for statistical computing. R Foundation for Statistical Computing, Disponível em: <http://www.Rproject.org>. Acesso em: 05 jun. 2017.

SCHNEIDER PR. 1997. Análise de regressão aplicada à engenharia florestal. Santa Maria: UFSM/CEPEF. 217p.

SCHOEPFER W. 1966. Automatisierrung des massen, Sorten und Wertberechnung stenender Waldbestande Schriftenreihe Bad. Berlin: WurttForstl. n.p.

SILVA F et al. 2011. Equações de afilamento para descrever o volume total do fuste de Pinus caribaea var. hondurensis na região do Triângulo Mineiro. Scientia Florestalis 39: 367-376.

SILVESTRE R et al. 2014. Equações Volumétricas em Povoamentos de Pinus taeda L. no Município de Lages-SC. Revista Nativa, Sinop 2: 1-5.

STEPKA TF et al. 2017. Modelos volumétricos e funções de afilamento para Pinus taeda L. na região Dos Campos Gerais, Paraná, Brasil. Espacios 38: 26-34.

TÉO SJ et al. 2013. Modelos de afilamento para Pinus elliottii em diferentes idades, na região de Caçador, SC. Revista Floresta 43: 439-452. 